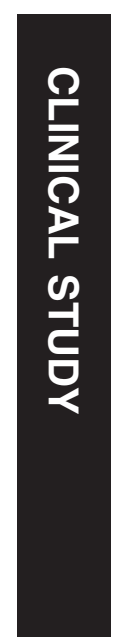

\title{
Evaluation of focal choroidal excavation in the macula using swept-source optical coherence tomography
}

${ }^{1}$ Department of Ophthalmology, Singapore National Eye Centre, Singapore

${ }^{2}$ Singapore Eye Research Institute, Singapore

${ }^{3}$ National University of Singapore, Yong Loo Lin School of Medicine, Singapore

Correspondence: BK Loh, Department of Ophthalmology (VitreoRetinal Service), Singapore National Eye Centre, 11 Third Hospital Avenue, Singapore 168751, Singapore

Tel: +6563227458

or + 65 97113390;

Fax: +62263395 .

E-mail: loh.boon.kwang@ snec.com.sg

Received: 8 October 2013 Accepted in revised form: 20 March 2014

Published online:

20 June 2014

This study has been presented at the Society of European Ophthalmology Meeting in Copenhagen on 8 June 2013.

\begin{abstract}
Purpose To evaluate imaging findings of patients with focal choroidal excavation (FCE) in the macula using swept-source optical coherence tomography (SS-OCT) and correlate it clinically.

Methods Prospective observational case series. Eleven consecutive patients (12 eyes) with FCE were described. Data on demographics and clinical presentation were collected and imaging findings (including color photography, fundus autofluorescence imaging, fluorescein angiography, indocyanine green angiography, spectraldomain optical coherence tomography, and SS-OCT) were analyzed.

Results The primary diagnosis was epiretinal membrane (two eyes), choroidal neovascularization (one eye), polypoidal choroidal vasculopathy (three eyes), central serous chorioretinopathy (one eye), and dry age-related macular degeneration (two eyes). Eleven out of 12 of the lesions were conforming. One presented with a nonconforming lesion that progressed to a conforming lesion. One eye had multiFCE and two had two overlapping choroidal excavations. Using the SS-OCT, we found the choroid to be thinned out at the area of FCE but sclera remained normal. The choroidal tissue beneath the FCE was abnormal, with high internal reflectivity and poor visualization of choroidal vessels. There was loss of contour of the outer choroidal boundary that appeared to be pulled inward by this abnormal choroidal tissue. A suprachoroidal space was noted beneath this choroidal tissue and the choroidal-scleral interface was smooth. Repeat SS-OCT 6 months after presentation showed the area of excavation to be stable in size.
\end{abstract}

FPM Lim¹, BK Loh ${ }^{1,2,3}$, CMG Cheung $1,2,3$ LS Lim ${ }^{1,2,3}$, CM Chan ${ }^{1,2,3}$ and DWK Wong 1,2,3
Conclusion FCE can be associated with epiretinal membrane, central serous chorioretinopathy, and age-related macular degeneration. The choroid was thinned out in the area of FCE.

Eye (2014) 28, 1088-1094; doi:10.1038/eye.2014.78; published online 20 June 2014

\section{Introduction}

Jampol et al $^{1}$ first described an unusual macular finding of excavation of the choroid on timedomain optical coherence tomography (OCT) in 2006 in a patient with good visual acuity and normal fundal examination. However, analysis of this finding was limited by time-domain OCT. Upon the introduction of the spectraldomain OCT (SD-OCT), several other cases of focal choroidal excavation (FCE) have been described. However, the understanding of these lesions, especially the etiology and clinical implication, remains limited. Wakabayashi et $a l^{2}$ described three cases of unilateral choroidal excavation without scleral involvement. In two of the cases, the outer retinal layer appears to conform to the choroidal defect without disruption or an optically clear space between the photoreceptor tips and the hyper-reflective retinal pigment epithelial band. The 3rd eye showed a disorganization of the photoreceptors and the retinal pigment epithelium (RPE). Margolis et $a l^{3}$ further reported 12 cases of FCE in 2011 in which one patient had bilateral FCE. In addition, they also found an association with central serous chorioretinopathy (CSC) and although most lesions remained stable, secondary choroidal neovascularization (CNV) may occur. More recently, Obata et al ${ }^{4}$ described the clinical features of 21 eyes and found FCE to 
be relatively stable even after long observation; however, during their follow-up, one eye developed CSC and one eye developed CNV.

Very recently, the swept-source OCT (SS-OCT) for the posterior imaging utilizing a wavelength of $1050 \mathrm{~nm}$ was introduced. Utilizing this $1050 \mathrm{~nm}$ wavelength, DRI OCT-1 can penetrate deeper compared with the current conventional OCT's with wavelength in the $850 \mathrm{~nm}$ range to better visualize the choroid and the sclera to enable us to understand the pathogenesis of this phenomenon. Ellabban et $a l^{5}$ used SS-OCT to study FCE in CSC eyes and found a prevalence of $7.8 \%$. Using the SS-OCT, they detected unusual choroidal tissue beneath the excavation which appeared to pull the outer boundary of the choroid inwards, hence postulating that FCE may have been formed from RPE retraction caused by focal scarring of choroidal connective tissue.

The aim of this study is to analyze the clinical and imaging findings of FCE using swept-source OCT in 11 patients (12 eyes) and postulate the etiology of this condition.

\section{Materials and methods}

This study followed the principles of the Declaration of Helsinki, with ethics approval obtained from the Singhealth centralized institutional review board.

Eleven consecutive patients (12 eyes) with FCE are described. Data on demographics, clinical presentation, and past medical history were collected. All patients underwent a comprehensive ophthalmic examination including best-corrected visual acuity, refraction, measurement of intraocular pressure, slitlamp biomicroscopy, and indirect ophthalmoscopy.

The choroidal excavation was detected by SD-OCT (Spectralis HRA-OCT; Heidelberg Engineering, Heidelberg, Germany), which acquires 40000 A-scans/sec.

\section{Swept-source optical coherence tomography}

We further analyzed it using the swept-source OCT (DRI OCT-1, Deep Range Imaging Optical Coherence Tomography, Topcon IMAGEnet systems, Tokyo, Japan) that can penetrate deeper compared with current conventional OCT. This new technology uses $1050 \mathrm{~nm}$ wavelength to provide deep wave imaging to better visualize the choroid and the sclera. It has a fast scanning speed of $100000 \mathrm{~A}-\mathrm{scans} / \mathrm{sec}$ and has increased scan coverage of the macula area to the disc, afforded by the $12-\mathrm{mm}$ wide scan. The scanning protocols used were the seven line raster scans and radial scans. The length, width, and depth of the intrachoroidal cavitation were measured with the built-in caliper function of the OCT software.

Focal choroidal excavation was defined as an area of macular choroidal excavation detected on an SD-OCT scan without evidence of a posterior staphyloma or scleral ectasia, in a patient lacking a history of trauma, posterior uveitis, retinal, or choroidal vascular disease, or before retinal or choroidal infection. Lesions were further subdivided into conforming and non-conforming. A conforming lesion was described as one with no separation of the photoreceptor tips and RPE, whereas a non-conforming lesion was one where the photoreceptors appear detached from the RPE with an intervening hyporeflective space. ${ }^{3}$

Autofluorescence (TRC-50DX, Topcon IMAGEnet systems, Spaide Autofluorescence Filter), fundus photography (TRC-50DX, Topcon IMAGEnet systems), fundal fluorescein angiography (FFA) (TRC-50DX, Topcon IMAGEnet systems), and indocyanine green (ICG) angiography (TRC-50DX, Topcon IMAGEnet systems) were also performed.

All patients were followed up for at least 6 months. Repeat fundal examination and SS-OCT were performed after 6 months from presentation to determine progression.

\section{Results}

\section{Demographics}

Eleven patients (12 eyes) were identified in which SSOCT scans revealed one or more focal areas of choroidal excavation. Patient demographics are summarized in Table 1 . The mean age of the patients was 57 year (range, 20-75). All patients were Chinese. Six out of 11 were male. Mean LogMAR best-corrected visual acuity was 0.28 (range, $0.10-0.50$ ). Eleven out of 12 eyes were myopic and mean spherical refractive error was-3.5D (range, 0-7.25D). One patient had bilateral FCE. Nine with associated primary diagnosis had visual symptoms of which three presented with metamorphopsia and six experienced blurring of vision. The two remaining patients that did not have a primary diagnosis at the FCE were asymptomatic. None of the patients reported a family history.

\section{Associated primary diagnosis}

In all eyes, the presence of choroidal excavation could not be determined on clinical examination or by color photography. However, ten out of 12 eyes had an associated primary diagnosis that was noted on clinical examination and color photography. The primary diagnosis was epiretinal membrane (ERM) (two eyes), $\mathrm{CNV}$ (one eye), polypoidal choroidal vasculopathy (PCV) (three eyes), CSC (one eye), and dry age-related macular degeneration (AMD) (two eyes). One patient (one eye) had an associated pigmented epithelial 
detachment and the last patient with bilateral focal excavation (two eyes) had no associated pathology.

\section{SS-OCT findings}

The FCE had an extrafoveal location in two eyes, a subfoveal location in four eyes and a juxtafoveal location in six eyes.

Out of the 12 eyes studied, 9 had discrete single lesions, 2 had 2 overlapping choroidal excavations, and the last patient had 2 distinct focal excavations in 1 eye.
Eleven out of 12 of the lesions detected on SS-OCT were conforming (defined as no separation of the photoreceptor tips and RPE). Eye two (Figure 1) who had an associated CSC presented with a non-conforming lesion (defined as one where the photoreceptors appear detached from the RPE with an intervening hyporeflective space) that progressed to a conforming lesion on repeat OCT 6 months later. This patient did not experience worsening of symptoms clinically.

The mean depth of the location was $142.4 \pm 114.8 \mu \mathrm{m}$ (range: $36-212 \mu \mathrm{m}$ ) and mean excavation width was

Table 1 Summary of the patient demographics, clinical features, and investigation findings for the patients in our case series

\begin{tabular}{|c|c|c|c|c|c|c|c|c|c|c|c|}
\hline $\begin{array}{l}\text { Eye } \\
\text { no }\end{array}$ & $\begin{array}{l}\text { Agel } \\
\text { Sex }\end{array}$ & $\begin{array}{l}\text { Refractive } \\
\text { error }\end{array}$ & $\begin{array}{l}\text { Presenting } \\
\text { symptom }\end{array}$ & $B C V A$ & Fundus appearance & Primary diagnosis & $\begin{array}{l}\text { Conforming/ } \\
\text { Non-conforming }\end{array}$ & $\begin{array}{l}\text { ELM and } \\
\text { IS/OS } \\
\text { junction }\end{array}$ & Location & $\begin{array}{c}\text { Depth } \\
(\mu \mathrm{m})\end{array}$ & $\begin{array}{l}\text { Width } \\
(\mu m)\end{array}$ \\
\hline 1 & $65 / F$ & -7.25 & Metamorphopsia & 0.2 & ERM & ERM & Conforming & Normal & Subfoveal & 61 & 540 \\
\hline 2 & $20 / \mathrm{M}$ & -4.5 & $\begin{array}{l}\text { Blurring of } \\
\text { vision; } \\
\text { metamorphopsia }\end{array}$ & 0.25 & RPE mottling & $\begin{array}{l}\text { Central serous } \\
\text { chorioretinopathy }\end{array}$ & Non-conforming & Normal & Subfoveal & 178 & 874 \\
\hline 3 & $61 / \mathrm{M}$ & -1.75 & $\begin{array}{l}\text { Blurring of } \\
\text { vision }\end{array}$ & 0.5 & $\begin{array}{l}\text { Parafoveal fusiform } \\
\text { lesion }\end{array}$ & $\begin{array}{l}\text { Choroidal } \\
\text { neovascularization }\end{array}$ & $\begin{array}{l}\text { Two conforming } \\
\text { choroidal } \\
\text { excavations. }\end{array}$ & Normal & Juxtafoveal & $\begin{array}{c}\text { Large } \\
\text { lesion } \\
212 \\
\text { Small } \\
\text { lesion } \\
36\end{array}$ & $\begin{array}{c}\text { Large } \\
\text { lesion } \\
514 \\
\text { Small } \\
\text { lesion } \\
178\end{array}$ \\
\hline 4 & $57 / \mathrm{M}$ & -3 & $\begin{array}{l}\text { Blurring of } \\
\text { vision }\end{array}$ & 0.5 & $\begin{array}{l}\text { PED, subretinal bleed, } \\
\text { subretinal fluid }\end{array}$ & $\begin{array}{l}\text { Polypoidal } \\
\text { choroidal } \\
\text { vasculopathy }\end{array}$ & Conforming & Disrupted & Juxtafoveal & 91 & 614 \\
\hline 5 & $55 / \mathrm{F}$ & -4 & Metamorphopsia & 0.2 & $\begin{array}{l}\text { PED and RPE atrophy } \\
\text { corresponding to area of } \\
\text { FCE }\end{array}$ & $\begin{array}{l}\text { Dry age-related } \\
\text { macular } \\
\text { degeneration }\end{array}$ & Conforming & Disrupted & Extrafoveal & 49 & 331 \\
\hline 6 & $67 / \mathrm{M}$ & -1 & $\begin{array}{l}\text { Blurring of } \\
\text { vision }\end{array}$ & 0.15 & Drusen & $\begin{array}{l}\text { Dry age-related } \\
\text { macular } \\
\text { degeneration }\end{array}$ & Conforming & Disrupted & Juxtafoveal & 114 & 720 \\
\hline 7 & $60 / \mathrm{M}$ & -4.5 & $\begin{array}{l}\text { Blurring of } \\
\text { vision }\end{array}$ & 0.1 & ERM & ERM & $\begin{array}{l}\text { Two overlapping } \\
\text { conforming } \\
\text { excavations }\end{array}$ & Normal & Juxtafoveal & 120 & 885 \\
\hline 8 & $70 / \mathrm{M}$ & -0.5 & $\begin{array}{l}\text { Blurring of } \\
\text { vision }\end{array}$ & 0.5 & PED and subretinal fluid & $\begin{array}{l}\text { Polypoidal } \\
\text { choroidal } \\
\text { vasculopathy }\end{array}$ & Conforming & Normal & Extrafoveal & 487 & 87 \\
\hline 9 & $75 / \mathrm{F}$ & - & $\begin{array}{l}\text { Blurring of } \\
\text { vision }\end{array}$ & 0.5 & $\begin{array}{l}\text { Orange nodule, } \\
\text { exudation, preretinal } \\
\text { hemorrhage }\end{array}$ & $\begin{array}{l}\text { Polypoidal } \\
\text { choroidal } \\
\text { vasculopathy }\end{array}$ & $\begin{array}{l}\text { Two overlapping } \\
\text { conforming } \\
\text { excavations }\end{array}$ & Disrupted & Subfoveal & 115 & 1212 \\
\hline 10 & $39 / \mathrm{F}$ & -2.5 & Asymptomatic & 0.2 & $\begin{array}{l}\text { PED ( }>1 \text { disc diameter } \\
\text { away from FCE) }\end{array}$ & PED & Conforming & Normal & Juxtafoveal & 100 & 446 \\
\hline${ }^{\mathrm{a}} 11$ & $56 / \mathrm{F}$ & -7.0 & Asymptomatic & 0.2 & RPE mottling & - & Conforming & Disrupted & Subfoveal & 147 & 776 \\
\hline${ }^{\mathrm{a}} 12$ & $56 / \mathrm{F}$ & -6.5 & Asymptomatic & 0.2 & RPE mottling & - & Conforming & Normal & Juxtafoveal & 141 & 514 \\
\hline
\end{tabular}

Abbreviations: ERM, epiretinal membrane; ELM, external limiting membrane; FCE, focal choroidal excavation; IS/OS, inner segment/outer segment; PED, pigment epithelial detachment; RPE, retinal pigment epithelium.

a Bilateral focal choroidal excavation in the same patient.

Figure 1 A 20-year-old Chinese male with a recent history of CSC had a non-conforming FCE demonstrated on OCT that progressed to a conforming type over 6 months. (a) Fundal examination of the left eye revealed retinal pigment epithelial layer changes and mottling. (b) Fundal autofluorescence shows fine granular hyperautofluorescence in the macula. (c) Spectral-domain OCT (SD-OCT) revealed a non-conforming FCE in the macula of the left eye at presentation. (d) Repeat SD-OCT after 6 months follow-up revealed progression of the typical non-conforming choroidal excavation to the conforming type of choroidal excavation. (e) FFA did not show any abnormal findings. (f) ICG angiography revealed a hypofluorescent spot that corresponds to the FCE (see arrow). (g) SS-OCT showing a thinned-out choroid at the area of the excavation (see arrow) and an intact external limiting membrane and inner-segment/ outer-segment junction. There was poor visualization of choroidal vasculature at the area of unusual thinned-out choroidal tissue and loss of contour of the outer choroidal boundary that appeared to be pulled inwards by this abnormal choroidal tissue. Note the presence of suprachoroidal space beneath this abnormal choroidal tissue. The choroidal-scleral interface was physiologically smooth, and the scleral was of normal curvature and thickness. 


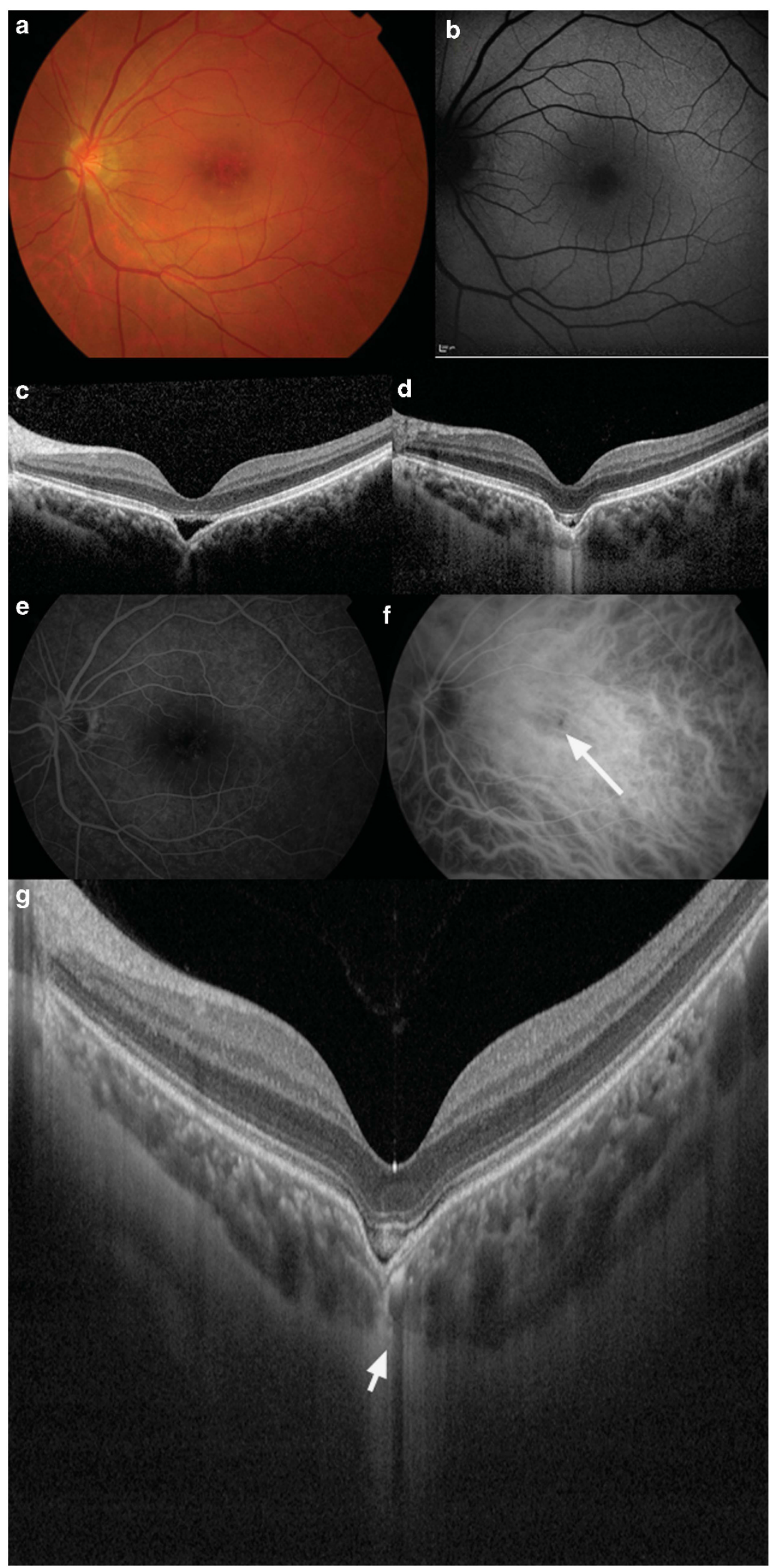


$591.6 \pm 307.0 \mu \mathrm{m}$ (range: $87-1212 \mu \mathrm{m}$ ). In all 12 eyes, the inner retinal layers appear physiologically normal above the FCE. Foveal contour remained well preserved even in subfoveal and juxtafoveal lesions. Six eyes had disruption of the external limiting membrane and innersegment/outer-segment junction at the area of FCE. Five of them had associated AMD (either the dry or wet form). Of the other five eyes with no disruption of the external limiting membrane and inner-segment/outersegment junction, only one had an associated PCV; however, the area of FCE was extrafoveal and $>1$ disc diameter away from the PCV.

The SS-OCT had allowed us to study the choroidal structures and scleral contour in detail. We found the choroid to be thinned out at the area of the FCE in all our patients, but the sclera contour and thickness remained normal. SS-OCT often showed an inner choroidal layer with medium-diameter blood vessels and an outermost choroidal layer with large-diameter vessels (Figure 2). In 10 out of 12 of the eyes, the thinned-out choroidal tissue just beneath the area of FCE was unusual, with high internal reflectivity and poor visualization of both the medium and large-diameter choroidal vessels. The large-diameter vessels were noted to be more affected. In addition, this unusual thinned-out choroidal tissue appeared to be contracted, with loss of contour of the outer choroidal boundary that appeared to be pulled inwards by this abnormal choroidal tissue. A suprachoroidal space was noted beneath this abnormal choroidal tissue and the choroidal-scleral interface was physiologically smooth in all 10 patients. The only two patients which did not demonstrate this phenomenon clearly on SS-OCT had relatively smaller lesions measuring less than $100 \mu \mathrm{m}$ in width and depth.

\section{FFA and ICG}

FFA and ICG were available in six eyes. An area of hypofluorescence on ICG angiography corresponding to the area of FCE was seen in one eye (Eye two). In the other five eyes, the areas of FCE appeared to be within the area of leakage secondary to PCV or CNV.

\section{Progression}

The mean follow-up duration was $18.2 \pm 10.1$ months (range: 6-24 months). In all of the 12 eyes, the area of FCE remained stable on OCT with no remarkable changes in measured width or depth. In the patient with CSC

(Figure 1), the photoreceptor tips reattached to the RPE, converting a non-conforming excavation to a conforming one. For the patients with CNV and PCV, clinical improvement was noted with treatment of the primary diagnosis; however, the conforming FCE persisted and

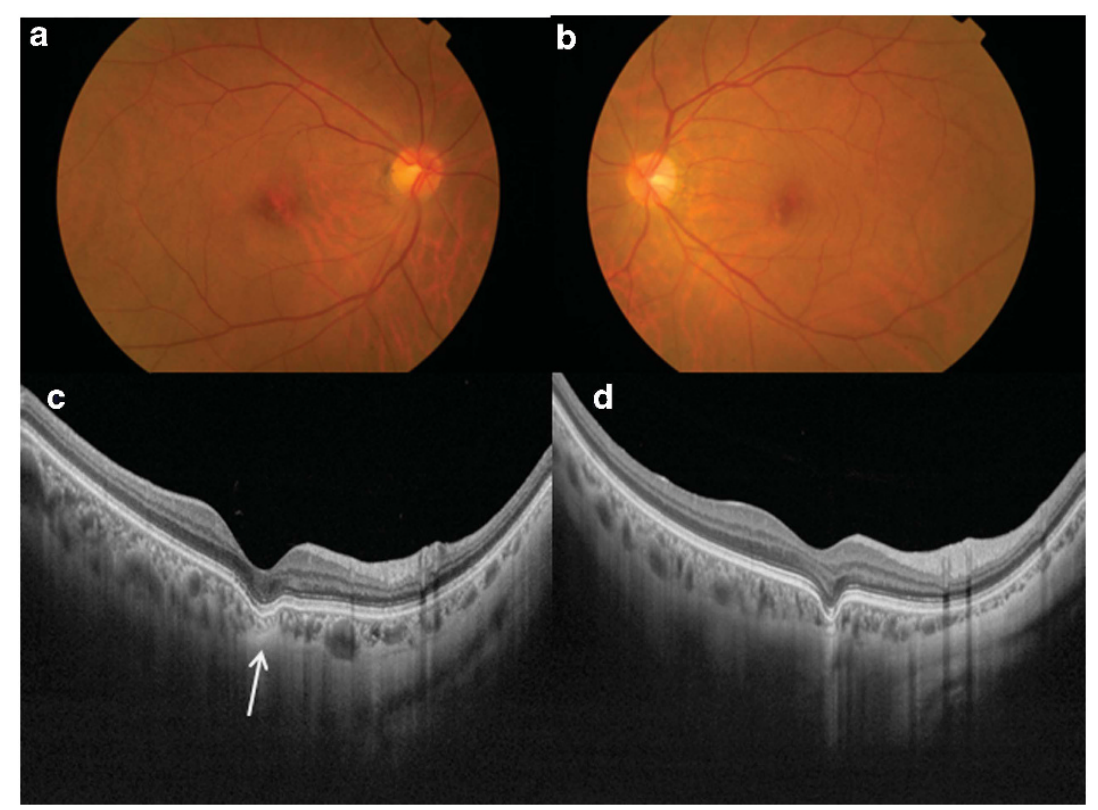

Figure 2 An asymptomatic 56-year-old Chinese female with conforming FCE in both eyes. Color fundus photographs of the right eye (a) and left eye (b) show RPE mottling. (c) Swept-source optical coherence tomography (SS-OCT) of the right posterior pole showing thinned-out choroidal tissue just beneath the area of FCE (arrow). This thinned-out choroidal tissue had high internal reflectivity and there was poor visualization of both the medium and large-diameter choroidal vessels. There is loss of contour of the outer choroidal boundary, which appeared to be pulled inwards by this abnormal choroidal tissue. A suprachoroidal space was noted beneath this abnormal choroidal tissue and the choroidal-scleral interface was smooth. (d) SS-OCT of the left posterior pole showing similar findings to the right eye. 
remained stable in size despite resolution of subretinal fluid on OCT. The patients with co-existing ERM, pigmented epithelial detachment, and dry age-related maculopathy remained stable clinically; the size of the conforming FCE also remained stable on OCT.

\section{Discussion}

We evaluated our case series of 12 eyes with FCE using SS-OCT and monitored for progression over time. The mean age of our patients was 57 years and age ranged from $20-75$ years. In two previous reports, the mean age was $45^{3}$ and $55^{4}$ which is similar to that of our study. Obata et al ${ }^{4}$ also reported a wide age range similar to ours in their study of 21 patients, suggesting that the etiology of this structure is multifactorial or formed and stabilized in early stage of life. Six out of 11 of our patients were male and other previous reports ${ }^{3,4}$ also did not find a gender predilection. Most were myopic with mean myopia of $-3.5 \mathrm{D}$, which is similar to two previous reports. ${ }^{3,4}$

Focal choroidal excavation has been reported to be associated with CSC, ERM, and subretinal neovascularization previously ${ }^{3-5}$ and more recently $\mathrm{CNV}^{6}$ and PCV. ${ }^{7}$ In our current study, we also found an association with all the above conditions as well as dry AMD. In most of the eyes with a co-existing pathology of $\mathrm{CSC}, \mathrm{PCV}, \mathrm{CNV}$, and dry AMD in our cohort, the area of FCE were located within the areas of pathology on fundal examination and FFA showed the location of FCE to be at the site of angiographic leakage. A recent study evaluating FCE in CSC patients, the site of FCE was also located in the area of angiographic leakage and choroidal hyperpermeability. ${ }^{5}$ As FCE is largely thought to be a choroidal abnormality and the pathogenesis of PCV, $\mathrm{CNV}$, and CSC are thought to be choroidal in nature, it has been suggested that FCE could predispose to the above conditions. ${ }^{5}$ However, it remains unproven that FCE is a platform for such choroidal pathologies to occur.

A small handful of patients have been reported to have visual symptoms of blurring of vision and metamorphopsia in previous studies. ${ }^{3,4}$ In our cohort, visual symptoms at presentation tend to be associated with co-existing AMD, previous CSC or ERM. The two patients in our cohort without a primary-associated diagnosis were asymptomatic. Previous reports have reported non-specific pigmentary changes and yellow spots correlating to the area of FCE on fundal examination, but none reported the direct visualization of the excavation clinically. ${ }^{1-3,5,8}$ Of our three patients with no co-existing pathology at the site of FCE, RPE mottling at the area of excavation was seen in two eyes, whereas no abnormality was detected in the remaining eye. In these eyes, observation over time did not show any worsening of symptoms or clinical appearance on fundal examination.

The main characteristics for FCE that we found in our patients using the SS-OCT were as follows: choroidal thinning at the area of the FCE; high internal reflectivity and poor visualization of choroidal vasculature at the area of unusual thinned-out choroidal tissue; loss of contour of the outer choroidal boundary that appeared to be pulled inwards by this abnormal choroidal tissue, and the presence of suprachoroidal space beneath this abnormal choroidal tissue. The choroidal-scleral interface was physiologically smooth and the scleral was of normal curvature and thickness in our cohort. These features of FCE demonstrated by OCT are similar to that reported by other studies. $3,5,9$ Our current study did not find any progression in size of FCE on SS-OCT over our follow-up period, which is consistent with what have been reported in other studies. ${ }^{3-5}$

The understanding of the pathogenesis and progression of FCE is still limited. Ellabban et al described the presence of unusual choroidal tissue beneath the excavation, and postulated that FCE may have formed from RPE retraction caused by scarring of choroidal connective tissue. Our study also demonstrated this characteristic thinned-out choroidal tissue as described above and agrees that it may represent scarring of choroidal connective tissue. However, the cause of this focal scarring remained largely unknown. The association of FCE with coexisting pathology, particularly CSC, CNV, and PCV, in our series and previous reports, suggest that before insult to focal area of the choroid could indeed be important for this focal scarring and hence excavation to develop. Once focal choroidal scarring has developed, the continued weakening of the choroid with age ${ }^{10}$ may lead to slow enlargement of the excavation, and conversion from nonconforming FCE into a conforming one (as we demonstrated in our case above). On the other hand, Margolis $\mathrm{et}_{\mathrm{al}}^{3}$ postulated that stress on the outer retina and eventual loss of the photoreceptor tips from the apical surface of the RPE may lead to conversion from conforming FCE to non-conforming FCE. The association of ERM and FCE is less understood. It has been reported that inflammatory mediators promote fibrovascular growth in the setting of secondary ERM formation. ${ }^{11}$ It could be possible that a prior intraocular inflammatory insult could result in eventual formation of focal choroidal scarring as well as ERM, resulting in the coexistence of both entities.

The strength of our study is the use of the latest sweptsource OCT (DRI OCT-1, Deep Range Imaging Optical Coherence Tomography, Topcon IMAGEnet systems) that can penetrate deeper compared with current conventional OCT. We are hence able to analyze the 
choroid and sclera in better detail in FCE using the SS-OCT compared with the SD-OCT. There has only been one report evaluating FCE with SS-OCT in CSC and our findings are consistent with it. ${ }^{5}$ However, we noted that there is poor visualization of the entire choroidal vasculature not just the large choroidal vessels as previously described; ${ }^{5}$ however, the absence of large choroidal vessels are more obvious due to its size.

The main limitations of our study include a small number of cases, a short follow-up duration and that it is carried out in a single institution. We only used line-scanning protocol of the SS-OCT, which may have a lower pick-up rate of FCE, compared with the three-dimensional scanning protocol as used by Ellabban et al..$^{5}$

In conclusion, FCE can be associated with ERM, central serous retinopathy, and AMD. The evaluation of this entity using the SS-OCT demonstrated choroidal thinning at the area of the excavation. More case accumulation and longer follow-up is needed to understand the condition better.

\section{Summary}

What was known before

- Focal choroidal excavation is a new entity that was first described by Jampol et al. ${ }^{1}$ It was defined as an area of macular choroidal excavation detected on an SD-OCT scan without evidence of a posterior staphyloma or scleral ectasia, in a patient lacking a history of trauma, posterior uveitis, retinal or choroidal vascular disease, or prior retinal or choroidal infection. As of today, etiology of FCE remains debated. In the very recent years, there are reported associations with central serous chorioretinopathy, dry AMD, subretinal neovascularization, and PCV.

\section{What this study adds}

- We found FCE to be associated with ERM, central serous chorioretinopathy, and AMD in our cohort. Using the SS-OCT, we were able to analyze FCE in depth. We found the choroid to be thinned out at the area of FCE but sclera remained normal. The choroidal tissue beneath the FCE was abnormal, with high internal reflectivity and poor visualization of choroidal vessels. There was a loss of contour of the outer choroidal boundary that appeared to be pulled inwards by this abnormal choroidal tissue. A suprachoroidal space was noted beneath this choroidal tissue and the choroidal-scleral interface was smooth. Repeat SS-OCT 6 months after presentation showed the area of excavation to be stable in size.

\section{Conflict of interest}

The authors declare no conflict of interest.

\section{Acknowledgements}

We would like to thank all the staff and allied health in the Singapore National Eye Centre for their support in this case series.This research received no specific grant from any funding agency in the public, commercial, or not-for-profit sectors.

\section{References}

1 Jampol LM, Shankle J, Schroeder R, Tornambe P, Spaide RF, Hee MR. Diagnostic and therapeutic challenges. Retina 2006; 26: 1072-1076.

2 Wakabayashi Y, Nishimura A, Higashide T, Ijiri S, Sugiyama K. Unilateral choroidal excavation in the macula detected by spectral-domain optical coherence tomography. Acta Ophthalmol 2010; 88: 87-91.

3 Margolis R, Mukkamala SK, Jampol LM, Spaide RF, Ober MD, Sorenson JA et al. The expanded spectrum of focal choroidal excavation. Arch Ophthalmol 2011; 129: 1320-1325.

4 Obata R, Takahashi H, Ueta T, Yuda K, Kure K, Yanagi Y. Tomographic and Angiographic characterisitcs of eyes with macular focal choroidal excavation. Retina 2013; 33 : 1201-1210.

5 Ellabban AA, Tsujikawa A, Matsumoto A, Yamashiro K, Oishi A, Ooto S et al. Three-dimensional tomographic features of dome-shaped macula by swept-source optical coherence tomography. Am J Ophthalmol 2013; 155: 320-328.

6 Katome T, Mitamura Y, Hotta F, Niki M, Naito T. Two cases of focal choroidal excavation setected by spectral-domain optical coherence tomography. Case Rep Ophthalmol 2012; 3: 96-103.

7 Kobayashi W, Abe T, Tamai H, Nakazawa T. Choroidal excavation with polypoidal choroidal vasculopathy: a case report. Clin Ophthalmol 2012; 6: 1373-1376.

8 Abe S, Yamamoto T, Kirii E, Yamashita H. Cup shaped choroidal excavation detected by optical coherence tomography: a case report. Retin Cases Brief Rep 2010; 4: 373-376.

9 Ohno-Matsui Kyoko, Akiba Masahiro, Moriyama Muka, Ishibashi Tatsuro, Hirakata Akito, Tokoro Takashi. Intrachoroidal cavitation in macular area of eyes with pathological myopia. Am J Ophthal 2012; 154: 382-393.

10 Margolis R, Spaide RF. A pilot study of enhanced depth imaging optical coherence tomography of the choroid in normal eyes. Am J Ophthalmol 2009; 147: 811-815.

11 Gilbert C, Hiscott P, Unger W, Grierson I, McLeod D. Inflammation and the formation of epiretinal membrane. Eye 1988; 2: 150-156. 